\title{
Editorial: Laser flare (cell) photometry: 20 years already
}

\author{
Carl P. Herbort $\cdot$ Ilknur Tugal-Tutkun
}

Received: 6 May 2010/Accepted: 3 June 2010/Published online: 1 July 2010

(C) Springer Science+Business Media B.V. 2010

Two decades ago, Sawa et al. [1] published an article entitled "New quantitative method to determine protein concentration and cell number in the aqueous in vivo" in the Japanese Journal of Ophthalmology. This work represented a milestone in the evolution of measurement of intra-ocular inflammation. The laser flare (cell) photometer $(\mathrm{LF}(\mathrm{C}) \mathrm{P})$ became commercially available in 1989-1990 and this technology promoted flare from a qualitative parameter to the only quantitative modality we have to measure intraocular inflammation.

In 1954, the first grading system of anterior intraocular inflammation using slit-lamp observation of the aqueous humor was proposed by a group of clinicians from the Proctor Foundation and had the merit of standardizing, at least in theory, inflammatory levels in the anterior chamber [2]. Although the system was supposed to set precise standards, it left a lot of space, especially as far as flare was concerned, for interobserver and intra-observer variation, as it was a

C. P. Herbort ( $\square)$

Inflammatory and Retinal Eye Diseases, Centre for Ophthalmic Specialized Care (COS), Clinique

Montchoisi, Lausanne, Switzerland

e-mail: carl.herb@bluewin.ch

C. P. Herbort

University of Lausanne, Lausanne, Switzerland

I. Tugal-Tutkun

Department of Ophthalmology, Istanbul Faculty

of Medicine, Istanbul University, Istanbul, Turkey subjective system based on individual interpretation of slit-lamp findings. As far as aqueous cells were concerned, the system was somewhat more precise as the counting of cells with the slit-lamp is possible to a certain extent, but it remained, nevertheless, semiquantitative at best. In 2004, uveitis specialists from around the world convened in Baltimore following the invitation of a group of American clinicians to decide on a new standardization system for uveitis nomenclature (SUN workshop) and, despite the fact that LFP had been on the market for more than 10 years and its sensitivity and reliability had been proven without question, the SUN workshop took over the Proctor Criteria almost unchanged for the assessment of intraocular inflammation [3].

Laser flare photometry (LFP) basically relies on the same principles as slit-lamp evaluation of flare. As for slit-lamp flare measurement, there is an incoming light beam and the quantity of diffracted light, proportional to the amount of cloudy matter in the aqueous humor (proteins), is measured. However the non-quantifiable polychromatic incident beam of the slit-lamp has been replaced by a quantifiable laser beam in LFP and the measuring device, the subjective human eye, is replaced by an objective photomultiplier-photodetector. Many studies were performed to show the immense superiority of this quantitative and objective method. In this issue a review article on LFP details the different studies that have been performed over the years [4].

Laser flare photometry was, at first, thought to be especially suited for clinical studies on intra-ocular 
inflammation and indeed it was used in innumerable studies. It allowed the evaluation of the anti-inflammatory power of numerous topically and systematically administered substances and enabled comparisons to be made between the substances. A selection of the numerous studies performed can be found in the review article in this issue [4].

However, it is in everyday uveitis practice that LFP technology has proven most useful. LFP-measured flare has become the only quantifiable parameter for most cases of intra-ocular inflammation, in both anterior and posterior segment inflammation, as long as in the latter there is a minimum of associated anterior chamber flare [5]. It was shown that LFP is not only essential in the assessment and follow-up of acute inflammation but also in chronic disruption of the blood-aqueous barrier. Even in the absence of cells, LFP can detect active and treatable inflammation, as was shown in a group of patients with juvenile idiopathic arthritis-associated uveitis, where a significant flare reduction after introduction of maximal therapy was demonstrated [6]. In this issue a clinical research article shows that LFP-measured flare is superior to slit-lamp cell count for the followup of inflammation in acute cases of HLA-B27related uveitis [7]. In essence, LFP is to uveitis what tonometry is to glaucoma: it allows measurement of the level of intra-ocular inflammation at any given time point. An interesting article in this issue also shows that in uveitis it is possible to measure flare in either dilated or non-dilated pupils, as no significant difference was found [8]; however, for technical reasons it is easier to measure flare in dilation.

Today LFP is used in many foremost centres throughout Europe and other parts of the world. Besides Europe, where LFP was rapidly integrated for everyday practice in leading centres in France, Germany, Italy, UK, Scandinavia and Switzerland, clinical LFP studies have recently been performed in Turkey and China $[9,10]$. In this issue Yang and colleagues have followed the evolution of flare in Behçet's uveitis [11].

Although LFCP allows the quantification of cells or particles in the aqueous humor, it is clinically less relevant. In clinical practice, the whole anterior chamber is examined for the presence of cells using the slit-lamp. The instrument, however, only evaluates a small volume, which makes it less reliable for cells as the distribution of cells in the anterior chamber is less diffuse and uniform than proteins. This drawback, present in the FC-1000 model, has been attenuated in the FC-2000 model which uses a larger measurement window. Nevertheless, this method allows objective measurement and quantitative analysis of cellular or particle numbers for the purpose of clinical studies.

This editorial comment applies only to slit-lamp $\mathrm{LF}(\mathrm{C}) \mathrm{P}$ photometers including the Kowa-FC-1000, Kowa FC-2000 and Kowa FM-500 models, all extremely reliable instruments. Unfortunately, the only company producing this technology has abandoned the instrument models constructed on the principle of the slit-lamp and today only produces one non-slit-lamp-based model, the Kowa FM-600. Although the new type of instrument has been tested and compared to previous models in study conditions, in everyday uveitis practice this new instrument is less suitable as it is fully automatic and does not allow manual adjustments to orient the incident beam as can be done in the slit-lamp construction principle. Therefore, no measurement can be obtained with the Kowa FM-600 in many uveitic eyes, especially in the "difficult" eyes where LFP is most required. Moreover, first results in a European setting comparing the two types of instruments in everyday uveitis practice also show that the Kowa FM-600 seems to be less reliable, under-estimating flare in low flare conditions. It is difficult to understand why the manufacturers stopped producing extremely reliable models such as the FM-500 measuring flare and the FC-2000 measuring flare and cells.

When such fundamental revolutionary instruments bringing significant medical progress are put on the market, the responsibility of the manufacturers goes beyond purely commercial interests. They are responsible to the medical community and the patients to assure the continuity of such an indispensible technology. We hope that slit-lamp models for LFP will again be produced in the near future, leaving the nonslit-lamp models for studies where more standardized conditions prevail.

\section{References}

1. Sawa M, Tsurimaki Y, Tsuru T, Shimizu H et al (1988) New quantitative method to determine protein concentration and cell number in aqueous in vivo. Jpn J Ophthalmol 32:132-142 
2. Hogan MJ, Kimura SJ, Thygeson P (1959) Signs and symptoms of uveitis. I. Anterior uveitis. Am J Ophthalmol 47(no.5, pt. 2):155-170

3. SUN Working Group (2005) Standardization of uveitis nomenclature for reporting clinical data. Results of the First International Workshop. Am J Ophthalmol 140:509516

4. Tugal-Tutkun I, Herbort CP (2010) Laser flare photometry: a noninvasive, objective and quantitative method to measure intraocular inflammation. Int Ophthalmol 30. doi: 10.1007/s10792-009-9310-2

5. Herbort CP, Guex-Crosier Y, de Ancos E, Pittet N (1997) Use of laser flare photometry (LFP) to assess and monitor inflammation in uveitis. Ophthalmology 104:64-72

6. Herbort CP (2009) Laser flare photometry. In: Gupta A, Gupta V, Herbort CP, Khairallah M (eds) Uveitis: text and Imaging. Jaypee Brothers, New Delhi, pp 28-49

7. Bernasconi O, Papadia M, Herbort CP (2010) Sensitivity of laser flare photometry compared to slit-lamp cell evaluation to monitor anterior chamber inflammation in uveitis. Int Ophthalmol 30 (in press)

8. Ikeji F, Pavesio C, Bunce C, White E (2010) Quantitative assessment of the effects of pupillary dilatation on aqueous flare with chronic anterior uveitis using laser flare photometry. Int Ophthalmol 30. doi:10.1007/s10792-0109373-0

9. Fang W, Zhou H, Yang P (2008) Quantification of bloodaqueous barrier breakdown and anterior cells in VogtKoyanagi-Harada syndrome following immunotherapy. $\mathrm{Br}$ J Ophthalmol 92:182-185

10. Tugal-Tutkun I, Cingu K, Kir N et al (2008) A use of laser flare-cell photometry to quantify intraocular inflammation in patients with Behçet uveitis. Graefes Arch Clin Exp Ophthalmol 246:1169-1177

11. Yang P, Fang W, Huang X, Zhou H, Wang L, Jiang B (2010) Alterations of aqueous flare and cells detected by laser flare-cell photometry in patients with Behcet's disease. Int Ophthalmol 30. doi:10.1007/s10792-008-9229-z 